\title{
Relationships between Supergranular horizontal flow velocity, cell size and cell lifetime
}

\author{
U. Paniveni ${ }^{1}$, V. Krishan ${ }^{1}$, Jagdev Singh ${ }^{1}$ and R. Srikanth ${ }^{2}$ \\ ${ }^{1}$ Indian Institute of Astrophysics, Koramangala, Bangalore-34, \\ email: udayams@vsnl.com \\ ${ }^{2}$ Raman Research Institute, Sadashivanagar, Bangalore-560080 \\ email: srik@rri.res.in
}

Keywords. Sun: granulation - turbulence

Supergranules are observed in the high photosphere as large convection eddies with horizontal diverging flows from the cell centre and subsiding flows at the cell borders.

In this work, we analysed 33 hour data of full disc Dopplergram obtained on 28th and 29th June 1996 by the Michelson Doppler Interferometer (MDI) on board the solar and Heliospheric observatory (SOHO) (Scherrer et al. 1995).

The line-of-sight velocities in the dark/bright region of the cells are directly read off from the velocity scan. Among them the first three highest velocity read-outsand the last three least velocity read-outs were selected. The maximum cell velocity is then determined as the average of the former three values minus the average of the latter three. This furnishes a simple way to assign a peak horizontal flow velocity $v_{h}$ to a given cellthat is independent of large scale velocity gradients.

The 33 hour data is spread over 198 frames with a 10 minute interval between the consecutive frames. Only those cells which appear and disappear within the chosen period of 33 hours are considered. This excludes cells already present in the first frame and those still present in the last frame. Thus the selected cells were born a few frames after the first. A particular supergranular cell thus identified is tracked down the successive frames until it disappears completely in a particular frame. Lifetime is identified to be the time interval between its first appearance and final disappearance.

The profile of a visually identified cell was scanned as follows: we chose a fiducial $y$-direction on the cell and performed velocity profile scans along the $x$-direction for all the pixel positions on the $y$-axis. In each scan, the cell extent is taken as the sum of the base sizes of the juxtaposed 'crest' and 'trough' expected in the Dopplergrams. The sum of all these values at all $y$-levels multiplied by the pixel dimension gives the area of the cell. The square root of this is taken to represent the length scale of the cell. The true size is obtained from the measured size by correcting for the projection effects.

A power law of the form

$$
v_{h}=f L^{\alpha}
$$

was fit to the data using the least squares method. For $r=0.6$, we find $f=0.014 \pm 0.007$ and $\alpha=0.34 \pm 0.046$. Figure 1 clearly shows that the two parameters are well correlated with a correlation co-efficient of 0.55. In an earlier study (Krishan 1991), it was suggested that the granulation and supergranulation are the result of energy cascading processes in a turbulent medium. If we apply the Kolmogorov hypothesis for a turbulent medium to the solar convective motions, we expect that the supergranular horizontal velocity $v_{h}$ depends on its size $L$ as $v_{h}=$ $\epsilon^{1 / 3} L^{1 / 3}$, where $\epsilon$ is the energy injection rate. Comparing this to Eq.0.1 suggests the identification $f=\epsilon^{1 / 3}$. According to the turbulent convection theory $\epsilon=v^{2} / T$, where we identify the eddy turnover time $T$ with the lifetime of a supergranular cell and $v$ is a typical velocity (identified with $v_{h}$ here $)$. Thus $\epsilon=(0.5)^{2} /(24 \times 60 \times 60)=2.89 \times 10^{-6} \mathrm{~km}^{2} \mathrm{~s}^{-3}$ and we find $f \approx 10^{-2}$ which implies $\epsilon \equiv f^{3}=10^{-6} \mathrm{~km}^{2} \mathrm{~s}^{-3}$. 


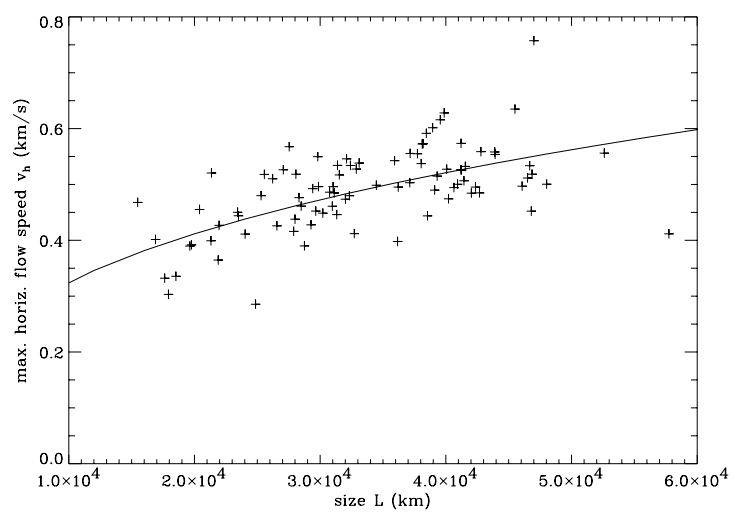

Figure 1. Plot of peak horizontal velocity $v_{h}$ against cell size $L$. The measured values are represented by the plusses. The line represents the least squares fit to $v_{h}=\epsilon^{1 / 3} \mathrm{~L}^{1 / 3}$.

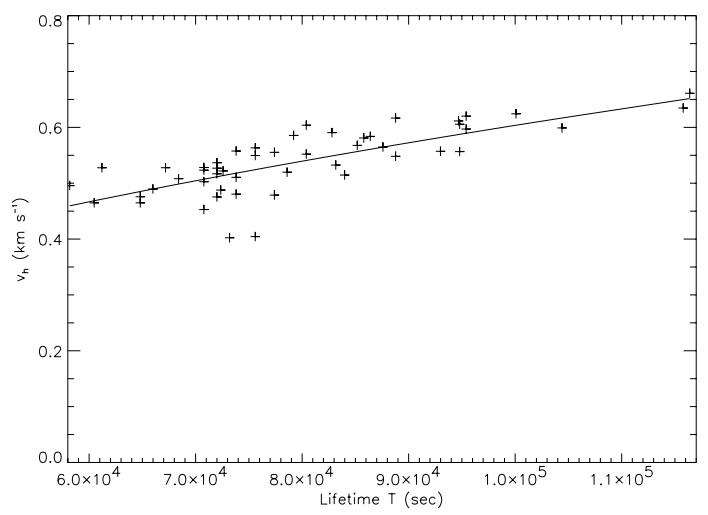

Figure 2. Plot of peak horizontal velocity $v_{h}$ against cell size $T$. The measured values are represented by plusses. The line represents the least squares fit to the equation $v_{h}=\epsilon^{1 / 2} T^{1 / 2}$

A power law of the form

$$
v_{h}=C T^{\alpha}
$$

was fit to the data using the least squares method. For $r=0.4$, we find $C=0.001$ where and $\alpha=0.51$. Figure 2 clearly shows that the two parameters are well correlated and the correlation coefficient between $v_{h}$ and $T^{1 / 2}$ is about 0.76 . Defining the eddy turnover time to be the lifetime of the cell, we can write $T=L / v_{h}$. Combining with the Kolmogorov spectrum, $v_{h}=\epsilon^{1 / 3} L^{1 / 3}$, we find, $v_{h}=\epsilon^{1 / 2} T^{1 / 2}$. Comparing this with Eq.0.2, we see that $C=\epsilon^{1 / 2}$. By our identification of eddy turn-over time with the lifetime of the cell, $\epsilon=0.5 \times 0.5 / 24 \times 60 \times 60 \approx 2.89 \times 10^{-6} \mathrm{~km}^{2} s^{-3}$ and hence $\epsilon=10^{-6} \mathrm{~km}^{2} \mathrm{~s}^{-3}$.

We thank Dr. P. H. Scherrer and the SOHO consortium for providing us with the MDI/SOI data.

\section{References}

Krishan, V., 1991, MNRAS, 250, 50

Scherrer, P. H., et al., 1995, Solar Physics, 162, 129

Singh, J., Nagabhushana, B. S., Babu, G. S. D. and Wahab Uddin, 1994, Solar Physics, 153, 157

Srikanth, R., Raju, K. P. and Singh, J., 2000, ApJ 534, 1008 